\title{
Polymyalgia Rheumatica After Influenza Vaccine
}

\author{
Mashal Salehia, b, Pavan Luckoora, Ed Marie Sibal-Gomez ${ }^{\mathrm{a}}$
}

\begin{abstract}
Influenza is an acute upper respiratory tract infection that occurs in epidemics almost every year, and is caused by influenza A and B viruses. It can cause serious complications including death especially in older adults and individuals with underlying health problems. Influenza vaccination is a very effective way of preventing influenza and is recommended by CDC. The common side effects of the vaccine vary from mild injection site reactions, to rhinorrhea, nasal congestion, headache and sore throat. On the other hand, there have been very rare reports of Guillain-Barre's syndrome and also some cases of polymyalgia rheumatica (PMR) after influenza vaccine. Our patient, an 86-year-old female, presented with bilateral shoulder and hip pain, and stiffness 2 weeks after she received her influenza vaccine, and both the patient and family were very adamant and upset that the problem was caused by the vaccination. Patient's erythrocyte sedimentation rate and C-reactive protein were elevated, a clinical diagnosis of PMR was made, and she was started on steroids to which she had a dramatic response. While we strongly recommend the administration of influenza vaccination, we also want to create awareness among the clinicians of this possibility, and proper education of the patients regarding this existence and the fact that if it happens at all, there is a treatment for it, and it should not discourage the patients from getting the vaccine in future. Also this association would need more studies in future.
\end{abstract}

Keywords: Polymyalgia rheumatica; Influenza vaccine; Inflammatory disease

\section{Introduction}

Polymyalgia rheumatica (PMR) is an inflammatory rheumatic condition characterized clinically by aching and morning stiffness in the shoulders, hip girdle, and neck [1]. It is almost exclusively a disease of adults over the age of 50 and unknown

Manuscript accepted for publication March 16, 2017

a Department of Medicine, NYC Health + Hospital/Harlem, Columbia University, New York, USA

${ }^{b}$ Corresponding Author: Mashal Salehi, Department of Medicine, NYC Health + Hospital/Harlem, Columbia University, 45 W 139th Street, Apt 17A, New York, NY 10037, USA. Email: Salehi.MashaL@yahoo.com

doi: https://doi.org/10.14740/jmc2788w eliciting factors. Symptoms are usually symmetric and onset can be startlingly abrupt [1]. The cause of PMR is unknown; both environmental and genetic factors appear to play role [1].

We report a case of a healthy elderly woman, previously well and independent in all ADLs who presented with a 2-week duration of symmetric muscle aches after receiving influenza vaccine.

\section{Case Report}

An 86-year-old Africa American female previously healthy, ambulatory with a cane and independent in her ALDs, presented with bilateral shoulder, and hip pain. Her symptoms started 2 weeks after receiving the flu vaccine. There was no history of similar symptoms in the past. On physical examination, patient was afebrile. There was decreased range of active and passive motion at the shoulder and hip joints, with normal muscle strength.

Laboratory workup was significant for elevation of erythrocyte sedimentation rate (ESR) $70 \mathrm{~mm} / \mathrm{h}$ and C-reactive protein (CRP) $2.67 \mathrm{mg} / \mathrm{dL}$, negative rheumatoid factor, and cyclic citrullinated peptide antibodies (anti-CCP) and ANA.

Patient was started on steroids, to which she had a dramatic response and was able to stand on her own feet the next day with free moment of her shoulder joints.

\section{Discussion}

Immunization with influenza vaccine is a widely accepted recommendation, a common practice in elderly and high-risk individuals, and usually very well tolerated [2]. Mild side effects of the vaccine are common while systemic symptoms as seen in our patient are very rare. Literature search yielded, very few cases have been reported where patients developed PMR after receiving flu vaccine. The actual phenomenon may not be correlated with the virus specificity but can be due to the vaccine adjuvants that are used for enhancing the immune response [3]. Although the incidence rate of this post-vaccination phenomena is unknown, the possibility that it could occur in the elderly should be considered, and needs further studies.

\section{Conclusion}

We strongly recommend influenza vaccination for all elderly 
patients.

We want to report this case to create awareness among clinicians of this possibility, that exist between PMR and influenza vaccine, and to let their patients know of a such possibility, and the fact that it can be treated effectively if one happens at all. This will not create the wrong impression of a serious side effect after vaccination in the elderly population and their families, and will not discourage them from having the vaccinations in future. This will also need further future studies.

\section{Financial Support}

No source of financial support in the form of any grants, equipment or drugs.

\section{Conflicts of Interest}

The authors declare that there are no conflicts of interest regarding the publication of this paper.

\section{Author Contributions}

All the authors certify that they have participated sufficiently in the intellectual content, analysis of date and that each one has reviewed the final version of the manuscript and approved it for publication.

\section{References}

1. Epidemiology of polymyalgia rheumatica in Olmsted County, Minnesota, 1970-1999.

2. Giant cell arteritis and polymyalgia rheumatica after influenza vaccination: report of 10 cases and review of the literature. Journals.sagepub.com/doi/ abs/10.1177/0961203311430222.

3. Soriano A, Verrecchia E, Marinaro A, Giovinale M, Fonnesu C, Landolfi R, Manna R. Giant cell arteritis and polymyalgia rheumatica after influenza vaccination: report of 10 cases and review of the literature. Lupus. 2012;21(2):153-157. 